\title{
Crystalography, morphology and optical properties of silver nanoparticles synthesized by extract of black pepper as a green method
}

\author{
Seyed Hamze Adnani Sadat ${ }^{*}$, Masoud Delgosha* \\ Department of Physics, University of Sistan and Baluchestan, 98135-674 Zahedan, Iran \\ Email address: \\ Hamze_adnani@yahoo.com (S. H. A. Sadat),delgosha.masoud@gmail.com (M. Delgosha)
}

\section{To cite this article:}

Seyed Hamze Adnani Sadat, Masoud Delgosha. Crystalography, Morphology and Optical Properties of Silver Nanoparticles Synthesized by Extract of Black Pepper as a Green Method. Optics. Vol. 3, No. 3, 2014, pp. 19-23. doi: 10.11648/j.optics.20140303.11

\begin{abstract}
An important aspect of nanotechnology is the development of reliable methods for the synthesis of nanomaterials in vitro that in view of the size, composition and shape can be controlled. In recent years, with increasing environmental problems, many due to green chemistry and attempts for the synthesis of nanomaterials through the environmental clean methods increased. The use of biological organisms for the clean synthesis of nanomaterials is important. In this article we report the synthesis of silver nanoparticles from silver nitrate solution by extract of black pepper from silver nitrate aqueous solution in various environment $\mathrm{pH}, 9,10,12$ and 13. Then crystalline structure, morphology and optical properties of these nanoparticles is presented. Prepared samples have been characterized by X-ray diffraction (XRD), UV-Visible Spectrometer (UV-vis) and Scattering Electron Microscope (SEM).
\end{abstract}

Keywords: Silver, Nanoparticles, Bological Synthesis

\section{Introduction}

Nanoparticles (NPs) are defined as having one dimension $100 \mathrm{~nm}$ or less in size and due to their large surface area they tend to react differently than larger particles of the same composition, allowing them to be utilized in novel applications [1]. Among all nanomaterials, silver nanoparticles (AgNPs) are unique as they are used in vast arrays of applications including medicine, food and consumer products [2]. Synthesis of such variety of AgNPs is possible by thermal decomposition [3], laser ablation [4], ultrasound [5] and aqueous chemical reduction method [6]. In order to minimise the risk of environmental hazards and to ensure economic sustainability by reducing production costs, there is a growing need to develop eco-friendly protocols for the nanoparticles synthesis employing green chemistry approaches using cheaply available, renewable natural resources [2]. One of them is synthesis of nanoparticles by biological process which implemented to develop safe, costeffective and environmentally friendly technologies [7]. Very recently green silver nanoparticles have been synthesized using various natural products like green tea (Camellia sinensis) [8], neem (Azadirachta indica) leaf broth [9], natural rubber [10], starch [11], aloe vera plant extract [12], lemongrass leaves extract $[13,14]$ leguminous shrub (Sesbania drummondii) [15], etc. Black pepper broth is known to contain several biomolecules such as alkaloids, proteins, polysaccharides, amino acids and vitamins. These biomolecules could be used as bioreductants to react with metal ions and they could also be used as scaffolds/template to direct the formation of nanoparticles in solution. The mechanism responsible for the reduction may postulate as follow: the silver ions were trapped on the surface of proteins/or predominated alkaloid piperine in the extract via electrostatic interactions. This stage was the recognition process. The silver ions were then reduced by the proteins leading to changes in their secondary structure and the formation of silver nuclei. The silver nuclei subsequently grew by the further reduction of silver ions and their accumulation of the nucle.

\section{Experimental}

\subsection{Extraction of Black Pepper}

For extraction, $20 \mathrm{~g}$ black pepper seeds washed thoroughly with distilled water and air-dried. After that powdered the 
seeds and boiled in $200 \mathrm{ml}$ distilled water for $10 \mathrm{~min}$. The cooled filtrate was adjusted at $\mathrm{pH} 14$ by adding $\mathrm{NaOH}$.

\subsection{Synthesis of Silver Nanoparticles}

The chemicals and reagents were used with high quality and purity (Merck, Germany).

Took $100 \mathrm{ml}$ of AgNO3 aqueous solution in a conical flask and adjusted at $\mathrm{pH} 9,10,12$ and 13 by adding extract drop by drop. Put the above solution on magnetic stirrer for $2 \mathrm{~h}$ and centrifuged at $3000 \mathrm{rpm}$ for $10 \mathrm{~min}$, supernatant decanted off and residue collected. Residue was washed three times with double distilled water followed by ethanol. Finally, residue kept in oven at $80 \circ \mathrm{C}$ for $10 \mathrm{~h}$.

\section{Results and Discussion}

Fig. 1 shows XRD patterns of the dried samples using $\mathrm{CuK} \alpha$ radiation $(0.154 \mathrm{~nm})$ for $2 \theta$ ranging from $20^{\circ}$ to $80^{\circ}$. The XRD patterns confirms the face-centered-cubic (fcc) lattice (JCPDS file no. 087-0718) with strong diffraction peaks at 38, 44, 64 and 77 degrees of $2 \theta$ which corresponds to (1 111$),\left(\begin{array}{lll}2 & 0 & 0\end{array}\right),\left(\begin{array}{lll}2 & 2 & 0\end{array}\right)$ and ( $\left.\begin{array}{lll}3 & 1 & 1\end{array}\right)$ crystal planes.
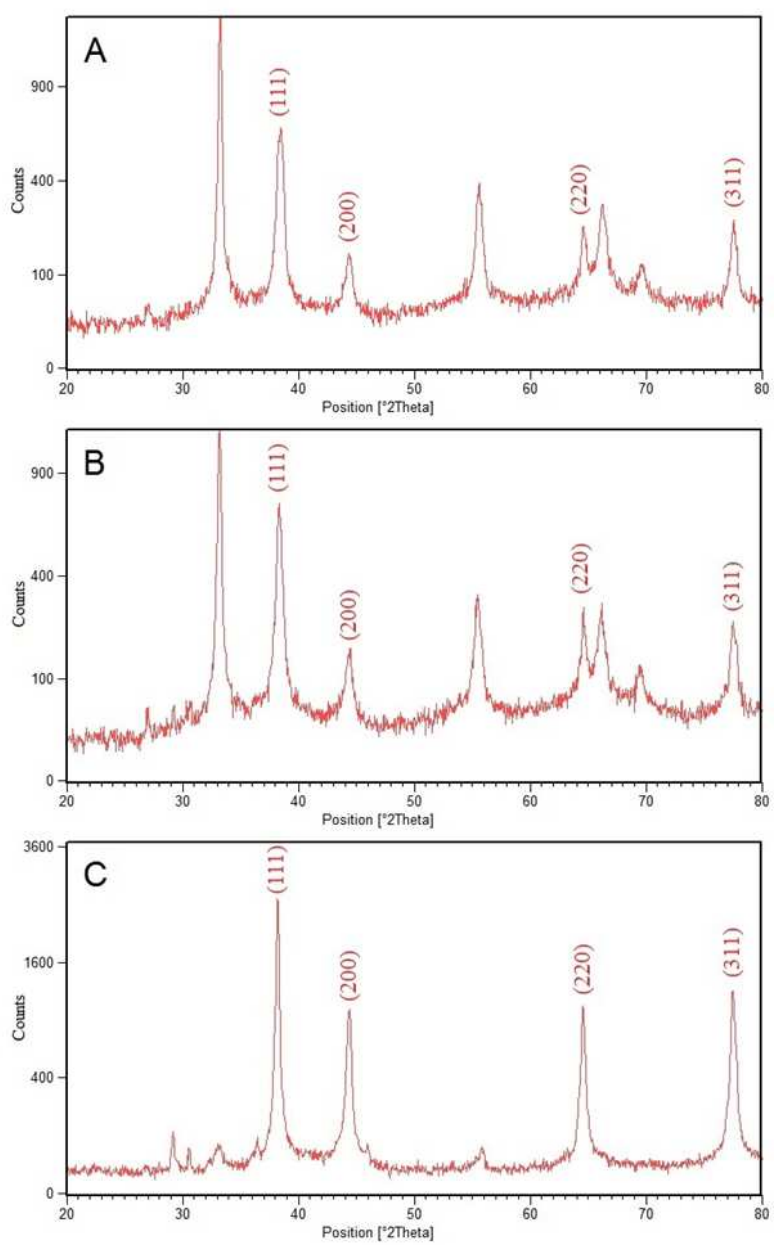

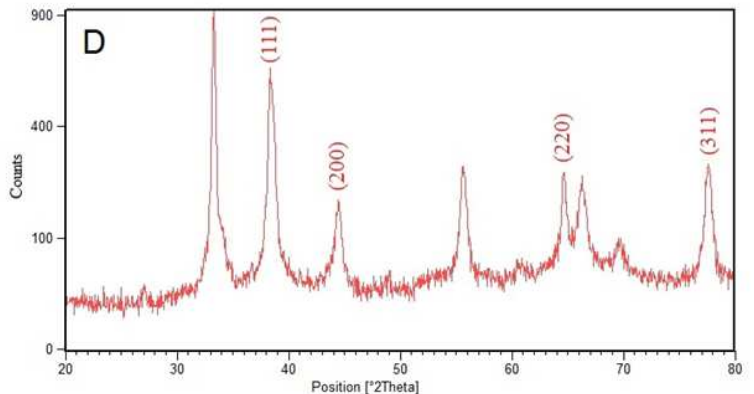

Fig 1. XRD patterns of samples $A, B, C$ and $D$ that synthesis respectively in pH 9, 10, 12 and 13

Fig. 2 shows XRD pattern of the dried sample that synthesis in dark environment. Pattern of this sample and D sample after heating shown in Fig. 3. There was no significant change in the peak position of silver nanoparticles in this patterns compared with Fig. 1.

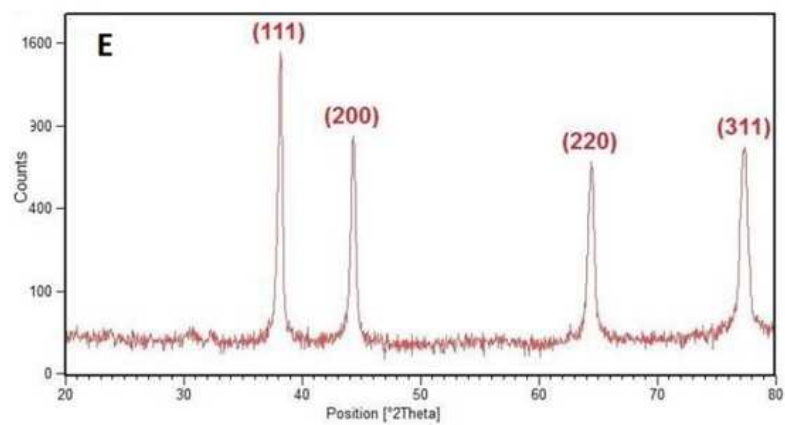

Fig 2. XRD pattern of sample that synthesis in dark environment
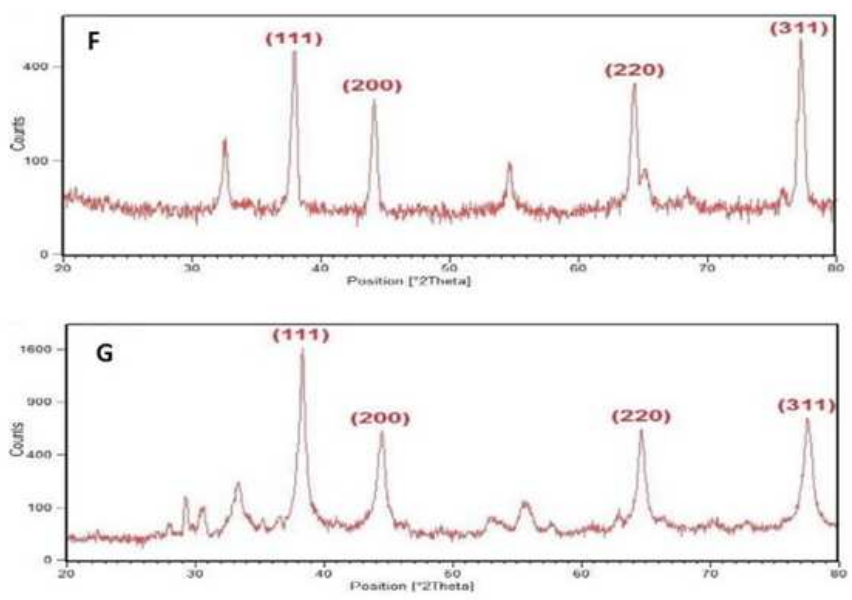

Fig 3. $F$ and $G, X R D$ pattern of respectively $E$ and $D$ sample after heating

Fig. 4 shows the Scattering Electron Microscope (SEM) images of the AgNPs. The nanoparticles formed had spherical shape of varied size. There was no marked difference in the shape. The biosynthesized AgNPs ranged in size between 20 and $60 \mathrm{~nm}$, this size range can be applied in areas of non-linear optics and solar cells, where very small nano-silver is required. 

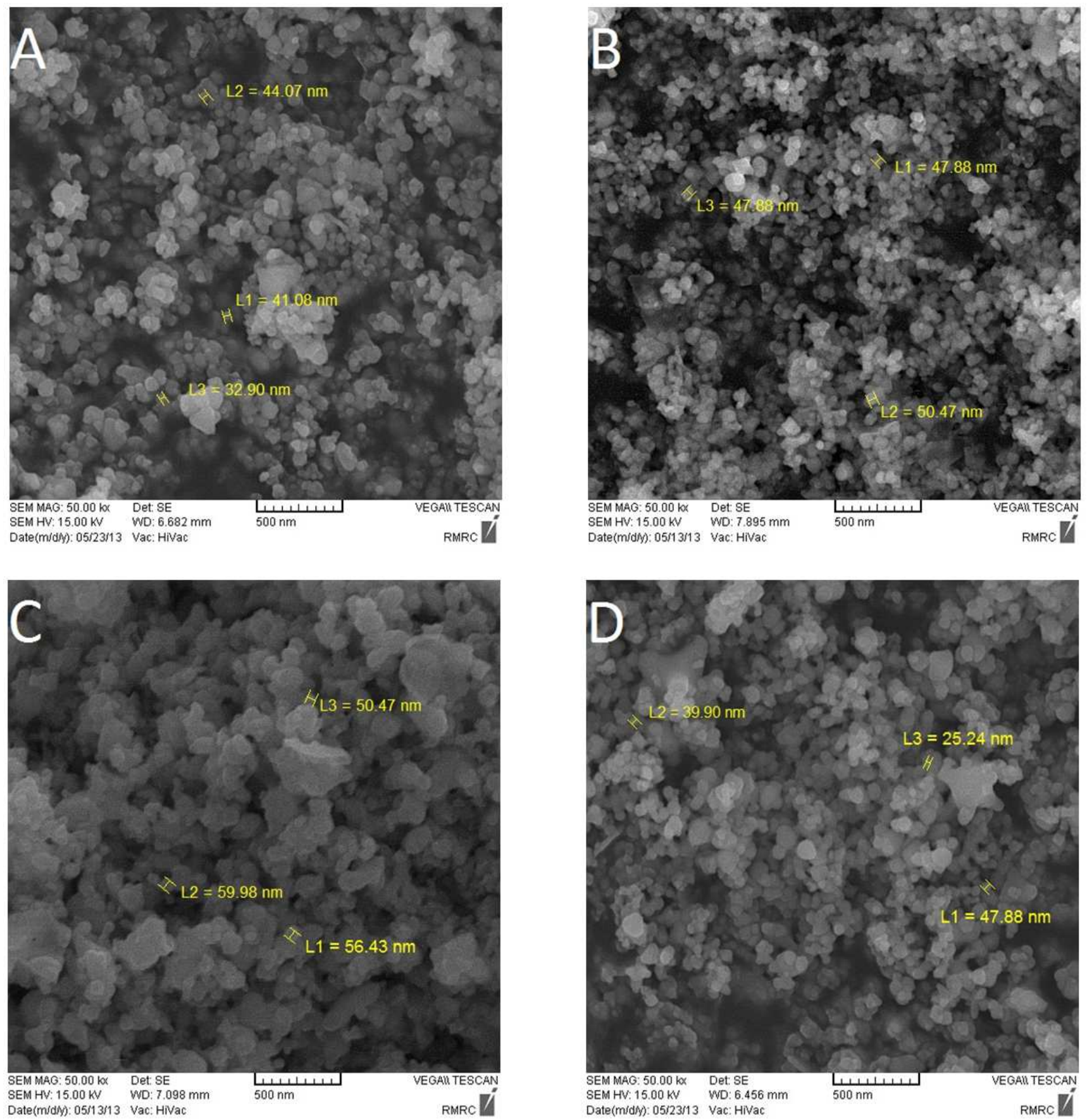

Fig 4. SEM images of samples $A, B, C$ and $D$ that synthesis respectively in $p H$ 9, 10, 12 and 13

Silver nanoparticles absorb radiation in the visible region of the electromagnetic spectrum due to the surface plasmon resonance (SPR) transition. Measurements were carried out on UV-visible spectrophotometer T80. The UV-vis absorption spectrum of the silver nanoparticles is shown in Fig. 5. In all these spectra, there are no peaks located around 335 and $560 \mathrm{~nm}$, indicating the complete absence of nanoparticle aggregation [16, 17]. UV-vis confirmed the existence of $\mathrm{Ag}$ particles because it is known from the reports in literature that spherical AgNPs display an UV-vis absorption peak in the region 400-450 nm. Although all preparations lead to the formation of silver nanoparticle but (according to Mie theory [18]) the different spectra suggest differences in particles sizes.

In Fig. 6 spectrum (E) shows the black pepper broth with $\mathrm{NaOH}$ treated (maintained at $\mathrm{pH} 14$ ), suggesting many peaks at UV wavelengths suggesting the basic amino acids or alkaloids, they might play role for AgNPs formation. Whereas, spectrum (F) shows the black pepper broth without $\mathrm{NaOH}$ treated, no peak appeared. However, work is in progress for deciphering the exact biomolecules of black pepper for playing crucial role to establish systematic AgNPs formation mechanism. 


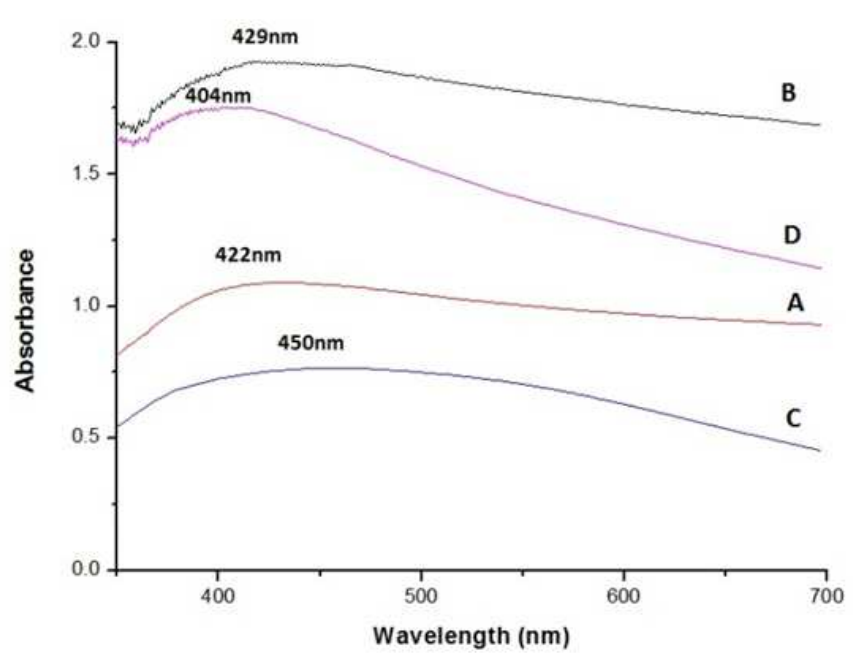

Fig 5. UV-vis absorption spectrum of samples $A, B, C$ and $D$ that synthesis respectively in $\mathrm{pH} 9,10,12$ and 13

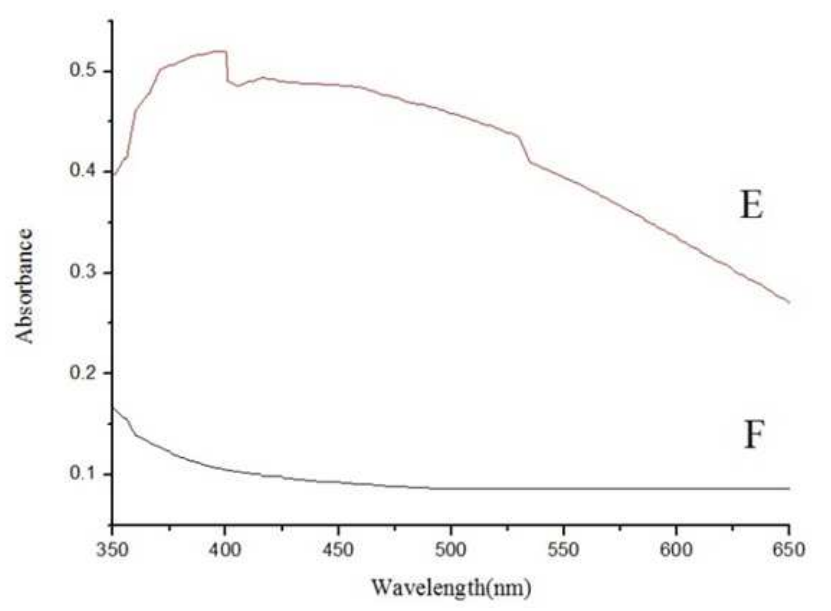

Fig 6. UV-vis absorption spectrum of black pepper broth (E) with $\mathrm{NaOH}$, (F) without $\mathrm{NaOH}$

\section{Conclusions}

A rapid, eco-friendly and novel synthesis route for preparing silver nanoparticles was carried out using black pepper extract as a reducing agent. The alkaloid piperine (or few basic amino acids or both) of the black pepper seed extract plays a leading role for the formation and stabilization of AgNPs, respectively.

The morphology of the NPs was determined from scanning electron microscopy (SEM). The formed nanoparticles were ranging in dimension 20 to $60 \mathrm{~nm}$ which was almost spherical in shape. The NPs was analyzed using UV-Vis spectroscopy and it points out to the formation of silver nanostructures with different sizes .Green synthesis method involved using black pepper extract in environmentally friendly universal solvent namely water, which would open new ventures in the application of formed silver nanoparticle. Our findings could be targeted for the promising potential applications including water purification, recording media, biosensing devices, nanoelectronic, and catalysis. Future research based on synthesis of size specific nanoparticle based on the optimization of reaction condition and study on the other properties of prepared silver nanoparticles such as antimicrobial, catalysis and etc would be an interesting area.

\section{References}

[1] Raudabaugh. D, Tzolov. M, Calabrese. J, Overton. B, Synthesis of Silver Nanoparticles by a Bryophilous Rhizoctoniaspecies, Nanomaterials and Nanotechnology, 2013, Vol. 3, Art. 2:2013.

[2] Meshram. Sh, Bonde. Sh, Gupta. I, Gade. A, Rai. M, Green synthesis of silver nanoparticles using white sugar, IET Nanobiotechnol., 2013, Vol. 7, Iss. 1, pp. 28-32.

[3] Navaladian. S, Viswanathan. B, Viswanath. P, Varadarajan. K, Thermal decomposition as route for silver nanoparticles, Nanoscaleres. Lett., 2007, Vol. 2, pp. 44-48.

[4] Zamiri. R., Azmi. Z., Darroudi. M., et al. Preparation of starch stabilized silver nanoparticles with spatial self-phase modulationproperties by laser ablation technique, Appl. Phys, 2011, Vol. 102, pp. 189-194.

[5] Vasileva. P, Donkova. B, Karadjova. I, Dushkin. C, Synthesis of starch-stabilized silver nanoparticles and their application as a surfaceplasmon resonance-based sensor of hydrogen peroxide, Coll. Surf. A,Physicochem. Eng. Aspects, 2011, Vol. 382, pp. 203-210.

[6] Kheybari. S, Samadi, N, Hosseini. V, Fazeli. A, Fazeli, R, Synthesis and antimicrobial effects of silver nanoparticles producedby chemical reduction method, DARU, 2010, Vol. 18, pp. $168-172$.

[7] Rupiasih. N, Aber. A, Gosavi. S, Vidyasagar. P, Green synthesis of silver nanoparticles using latex extract of Thevetia peruviana: a novel approach towards poisonous plant utilization, Journal of Physics: Conference Series423(2013).

[8] Vichis-Nestor. A, Sanches-Mendieta. V, Camacho-Lopez. M, Gomez-Espinosa. R, Camacho-Lopez. J, Arenas-Alatorre. A, Solventless synthesis and optical properties of $\mathrm{Au}$ and $\mathrm{Ag}$ nanoparticles using Camellia sinensis extract, Mater. Lett, 2008, Vol. 62,pp. 3103-3105.

[9] Shiv Shankar. S, Rai. A, Ahmad. A, Sastry. M, Rapid synthesis of $\mathrm{Au}$ cor-Ag shell nanoparticles using neem (Azadirachta indica) leaf broth. J. Colloid Interf. Sci. 275, 2004, pp. 496502.

[10] Abu Bakar. N, Ismail. J, Abu Bakar. M, Synthesis and characterization of silver nanoparticles in natural ribber, Mater. Phys , 2007, Vol. 104, pp. 276-283.

[11] Vigneshwaran. N, Nachane. R, Balasubramanya. R, Varadarajan. P, A novel one pot green synthesis of stable silver nanoparticles using soluble starch, Carbohydr. Res, 2006, Vol. 341, pp. 2012-2018.

[12] Chandran. S, Chaudhary. M, Pasricha. R, Ahmad.A, Sastry. M, Synthesis of gold nanotriangles and silver nanoparticles using aloevera extract, Biotechnol. Prog, 2006, Vol. 22, pp. 577-583.

[13] Shankar. S, Rai. A, Ankamwar. B, Singh. A, Ahmad. A, Sastry. M, Biological synthesis of triangular gold nanoprisms, Nat. Mater, 2004, Vol. 3, pp. 482-488. 
[14] Shankar. S, Rai. A, Ahmad. A, Sastry. M, Controlling the optical properties of lemongrass extract synthesized gold nanotriangles and potential application in infrared-absorbing optical coating, Chem. Mater, 2005, Vol. 17, pp. 566-572.

[15] Sharma. N, Sahi. S, Nath. S, Parsons. J, Gardea-Torresdey. J, Pal. T, Synthesis of plant-mediated gold nanoparticles and catalytic role of biomatrixembedded nanomaterials, Envention. Sci. Technol, 200, Vol. 41, pp. 5137-5142.

[16] Kora. A, Manjusha. R, Arunachalam. J, superior bactericidal activity of SDS capped silver nanoparticles . Synthesis and characterization, Materials Science and Engineering, 2009, Vol. 29, pp. 2104-2109.

[17] Mohan. Y, Raju. K., Sambasivudu, K., Singh. S, Sreedhar. B, Preparation of Acacia-stabilized silver nanoparticles: A green approach. Journal of Applied Polymer Science, 2007, Vol. 106, pp. 3375-3381.

[18] Guozhong. C, Nanostructures and Nanomaterials, Imperial Colleg Press, 2004, pp. 565-566. 